\title{
Quality of service performances of video and voice transmission in universal mobile telecommunications system network based on OPNET
}

\author{
Sameer A. S. Lafta1, Mohaned Mahdi Abdulkareem², Raed Khalid Ibrahim³, Marwah M. Kareem4, \\ Adnan Hussein Ali $^{5}$ \\ ${ }^{1}$ Middle Technical University, Technical Instructors Training Institute, Baghdad, Iraq \\ ${ }^{2}$ Ministry of communication, Baghdad, Iraq \\ ${ }^{3}$ Department of Medical instruments engineering techniques, Al-Farahidi University, Baghdad, Iraq \\ ${ }^{4}$ Computer Techniques Engineering, Al-Esraa College University, Baghdad, Iraq \\ ${ }^{5}$ Middle Technical University, Institute of Technology-Baghdad, Iraq
}

\begin{tabular}{|c|c|}
\hline Article Info & ABSTRACT \\
\hline Article history: & The universal mobile telecommunications system (UMTS) has distinct \\
\hline Received Jul 14, 2021 & $\begin{array}{l}\text { benefits in that it supports a wide range of quality of service }(\mathrm{QoS}) \text { criteria } \\
\text { that users require in order to fulfill their requirements. The transmission of }\end{array}$ \\
\hline Revised Sep 28, 2021 & video and audio in real-time applications places a high demand on the cellular \\
\hline Accepted Oct 1, 2021 & $\begin{array}{l}\text { network, therefore QoS is a major problem in these applications. The ability } \\
\text { to provide QoS in the UMTS backbone network necessitates an active QoS }\end{array}$ \\
\hline Keywords: & $\begin{array}{l}\text { mechanism in order to maintain the necessary level of convenience on UMTS } \\
\text { networks. For UMTS networks, investigation models for end-to-end QoS, }\end{array}$ \\
\hline OPNET & $\begin{array}{l}\text { total transmitted and received data, packet loss, and throughput providing } \\
\text { techniques are run and assessed and the simulation results are examined. }\end{array}$ \\
\hline QoS & According to the results, appropriate QoS adaption allows for specific voice \\
\hline Received and sent traffic data & and video transmission. Finally, by analyzing existing QoS parameters, the \\
\hline $\begin{array}{l}\text { Throughput } \\
\text { UMTS }\end{array}$ & QoS performance of 4G/UMTS networks may be improved. \\
\hline
\end{tabular}

Video and voice transmission

\begin{abstract}
The universal mobile telecommunications system (UMTS) has distinct benefits in that it supports a wide range of quality of service (QoS) criteria in order to fulfill their requirements. The transmission of network, therefore QoS is a major problem in these applications. The ability to provide QoS in the UMTS backbone network necessitates an active QoS mechanism in order to maintain the necessary level of convenience on UMTS total transmitted and received data, packet loss, and throughput providing techniques are run and assessed and the simulation results are examined. and video transmission. Finally, by analyzing existing QoS parameters, the
\end{abstract}

This is an open access article under the CC BY-SA license.

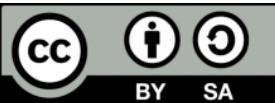

\section{Corresponding Author:}

Adnan Hussein Ali

Middle Technical University

Institute of Technology

Baghdad, Iraq

Email: aaddnnaann63@gmail.com

\section{INTRODUCTION}

As a consequence of the numerous advancements in wireless technology over the last decade, communications via technological means have gradually become the most popular. The rapid growth of these technologies has aided the improvements in meeting consumers' promises [1]. New cellular network inventions may be created in response to user requests. Due to the growing number of demand-driven apps, a variety of service kinds are now available to meet customer expectations. Against this backdrop, an effective quality of service (QoS) technique might be regarded important for helping and enhancing the quality of various services aimed at satisfying users' satisfaction [2].

The internet's technology focuses on recent innovations that have altered users' communication methods. Due to the rapid growth of wireless packet-switched networks, sending data over the Internet as an alternative to the public switched telephone network (PSTN) may be considered a better option in terms of user cost and service providers, resulting in an enormous increase in the application uses of voice over internet protocol (IP) networks [3]. The IP development of low-cost broadband access and based networks 
has sparked a lot of interest in voice over IP (VoIP) services [4]. To be really appealing, the VoIP service's QoS must be similar to that of traditional telephony [5]. It is important to enhance data transmission quality as the use of wireless networking grows. WLAN, WiMAX, and universal mobile telecommunication system (UMTS) are all wireless networks with their own set of advantages [6].

In recent years, mobile users have become more flexible with voice and video transmissions (VVT). This emphasizes the need of good speech quality at the receiver's end, as voice/video transmission via a single network will degrade speech quality at the recipient owing to factors such as coverage area, data rate, and movement. This challenge might be solved by integrating individual networks into a heterogeneous network.

The aim of this article is to assess the existing QoS performance of video and audio conferencing on a 4G/UMTS network using key performance indicators including packet loss, delay, and throughput. The output of the performance metrics will be depicted in a simulation model and graphs to realize and assess the performance of QoS 4G/UMTS wireless networks.

\section{RESEARCH METHOD METHODS and MATERIALS}

\subsection{Universal mobile telecommunication system}

Explaining UMTS, as one of the $3 \mathrm{G}$ mobile communication technologies, has been upgraded to include 4G technology. WCDMA International mobile communications with direct spread (IMT) is used as a front air to overlap in the most popular kind of UMTS [7]. This system, however, also contains a Timedivision multiple access (TDMA) and a synchronized access time to code division multiple access (TCDMA), which covers all IMT, CDMA, and TDD.

UMTS allows users to communicate easily regardless of their location, terminal type, technological choice, or transmission manner [8]. Another developing wireless technology is the development and deployment of UMTS to the $3 \mathrm{G}$ network. The UMTS is a comprehensive network system that gives users high mobility and coverage to fulfill their needs in a variety of settings, including the home, workplace, rural, and urban regions as shown in Figure 1 [9], [10]. Packet-based applications, such as real-time multimedia applications, are supported by the system.

The basic architecture of 4G UMTS was built on the GSM network [11], which is represented by: the USIM or UMTS SIM card in a GSM phone is backward compatible, and the SS7 MAP protocol of UMTS is based on the GSM MAP. For circuit and packet transmission, similar but modified protocols are utilized, and specific processes are needed during packet and circuit switching to guarantee seamless hard handovers.

Many features defined by the IMT, such as flexibility, compatibility with the current system, affordability, design modularity, and high-speed packet access (HSPA) are offered by 3G. For UMTS, it offers HSPA [12] like a mixture of HSDPA and HSUPA. The 3GPP is also described as Enhanced HSPA or HSPA + (3GPP Rel-7) and Rel-8 LTE, also referred to as evolved UMTS terrestrial radio access (EUTRA). $4 \mathrm{G}$ is being deployed currently [13].

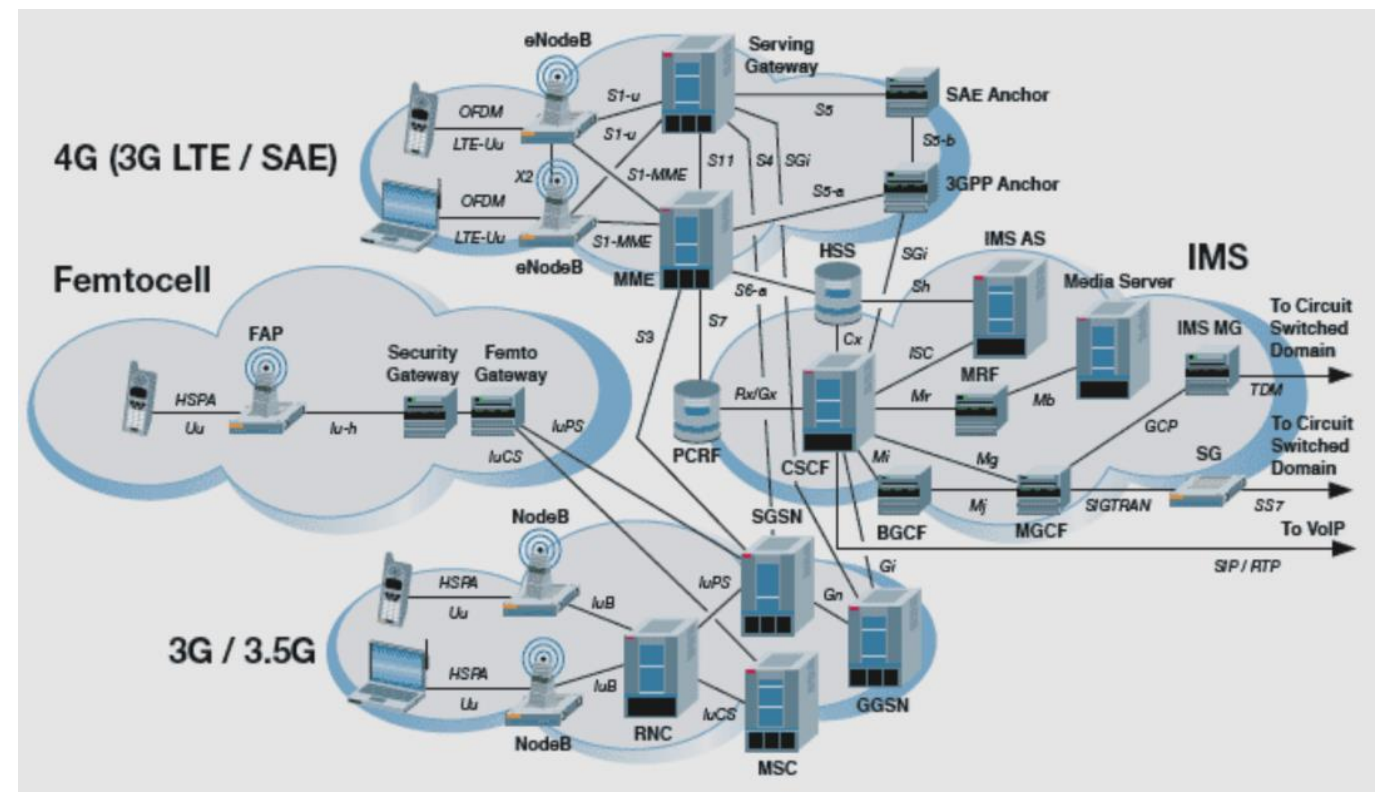

Figure 1. Sample $3 \mathrm{G}$ and $4 \mathrm{G}$ network 


\subsection{UMTS protocol architecture}

The architecture of the UMTS protocol for the propagation of data created by IP-based applications can be seen in Figure 2. The IP suites and the applications are located at the end-nodes [14]. Header compression functionality is provided by the PDCP while the RLC layer can function in either acknowledged transparent or unacknowledged mode. A dependable data transfer can be achieved over an error-prone radio interface using the acknowledged mode while the transparent and un-acknowledged modes cannot ensure data delivery [15]. The transparent mode is mainly developed for application in the UMTS circuit-switched mode where data passed unchanged through the RLC. The operation of the MAC layer can either be in the common or dedicated mode [16]. Operation in the dedicated mode requires allocation of dedicated physical channels by one user but in the common mode, the physical channels are shared by the users for data propagation. In addition to all the radio functions, the physical (PHY) layer also has signal distribution and processing capabilities, including, forward-error-correction, power control, and interleaving [17].

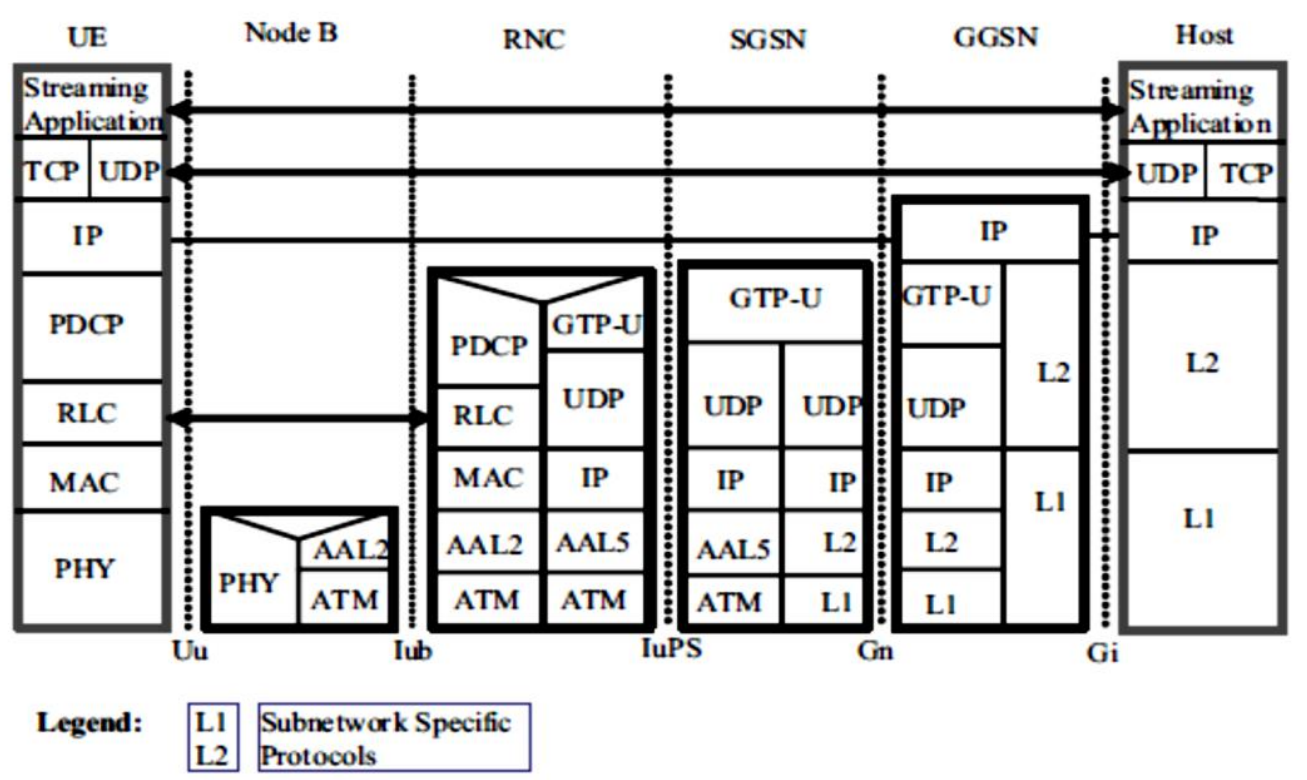

Figure 2. UMTS protocol architecture

\subsection{UMTS architecture}

A high-level perspective of the UMTS network framework is depicted in Figure 3; it is comprised of 6 major components (UTRAN, core network, circuit switched network, SS7 network, and the external packet data network) and the major interfaces between the entities [18].

The major components of the Core network are a circuit switched (CS) domain and a packet switched (PS) domain [19]. The CS provides voice and circuit-switched data services while PS provides packet-based services. A logical framework of the UMTS is presented in Figure 4, with the CS domain shown on the left and the PS domain on the right. For both domains, UTRAN is the radio access network; it is comprised of a set of RNS which consists of 2 major logical elements (Node B and RNC) [20]. The RNS is responsible for transmission and reception processes in cells where a broadcast channel serves a coverage area.

\subsection{UMTS network models}

MAC model: This model is implemented at the dedicated mode. The MAC layer requires a series of buffered blocks at the RLC layer. These buffered blocks are for transmission and submission as transport blocks to the PHY layer [21]. Here, each transport block is associated with an RLC block because, in the dedicated mode, no MAC header is required Figure 5. The transmission time interval (TTI) refers to the frequency required to facilitate the acceptance of the transport blocks from MAC by the PHY layer. The TTI values in the UMTS standard are 10, 20, 40, and $80 \mathrm{~ms}$ [22], [23]. 


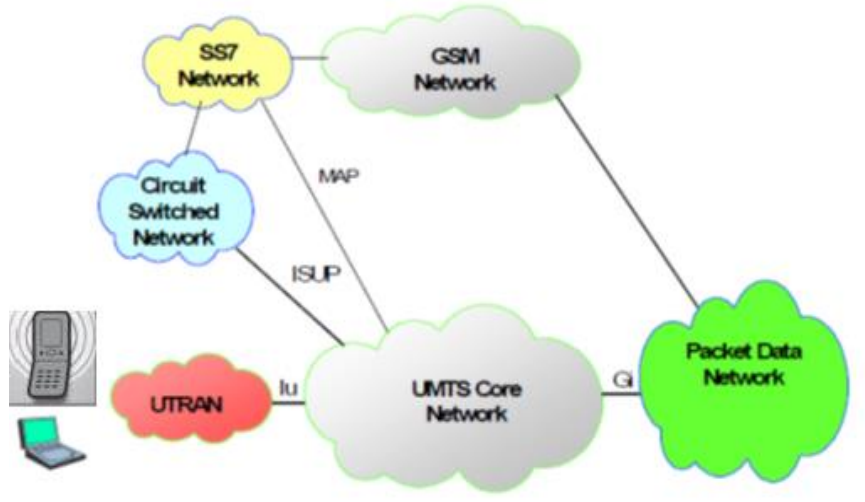

Figure 3. UMTS architecture

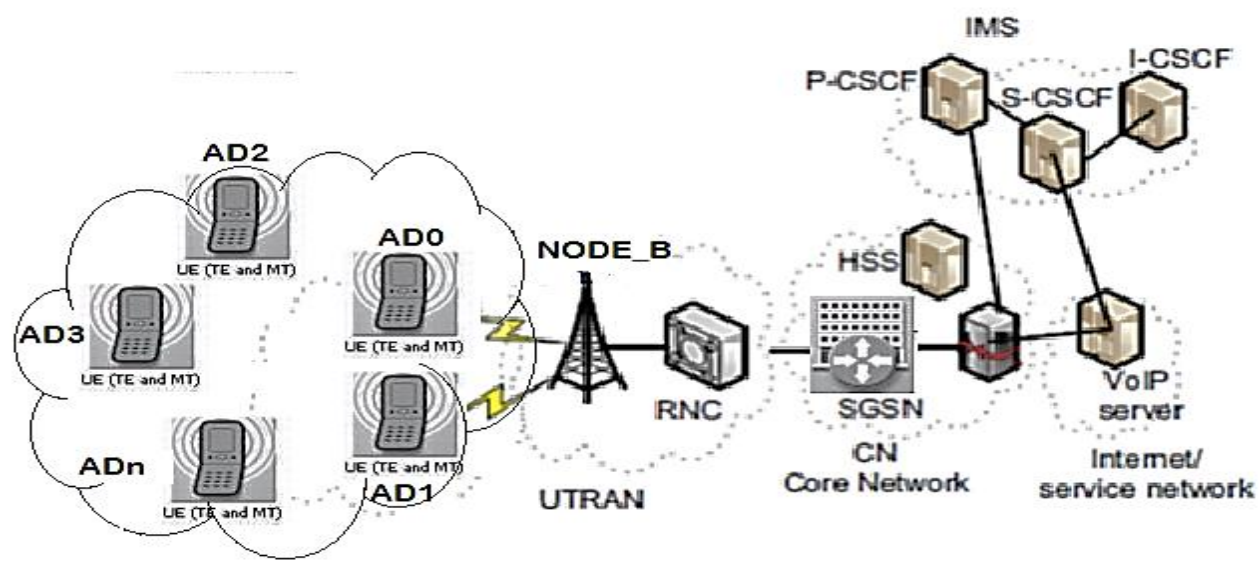

Figure 4. VoIP in UMTS

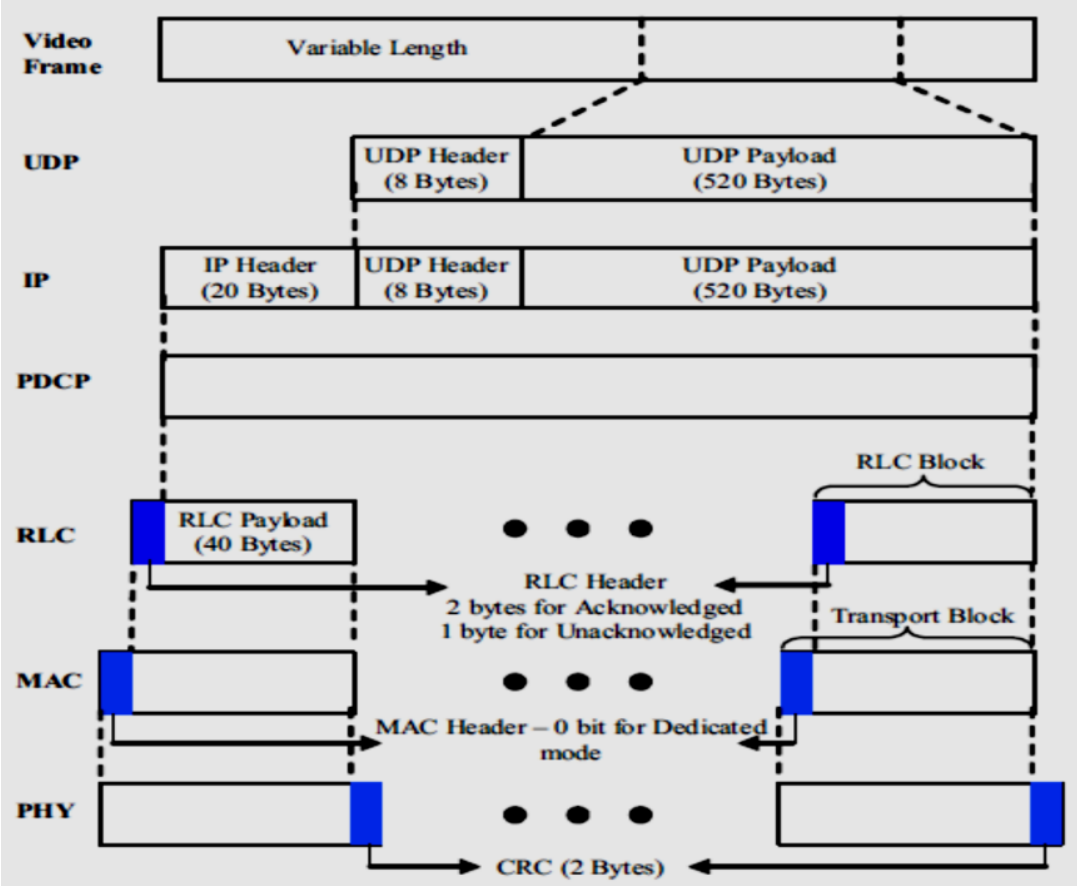

Figure 5. IP packet data transfer 
PHY model: This model is mainly for transport blocks propagation over the physical channels. The transport blocks for the MAC dedicated mode are propagated over the DPCH which ensures a fixed bit rate throughout the connection. The other functions of the PHY layer are not executed in the model [24], [25]. Being that the PHY layer forwards the transport block and the CRC error indicator to the MAC layer, the PHY layers' output can be characterized by the total transport block error probability, known as transport block error rate (TBLER) [26].

Figure 5 illustrates video transmission over the UMTS network. The UDP carry each encoded video frame over the IP protocol; however, video frames of larger size than the UDP segment size (520 bytes) are broken down. The IP packets are embedded into PDCP packets before sending them as a PDCP packet of comprising of IP packet of 548 bytes to the RLC. Then, the PDCP packet is fragmented into several fixedsized RLC blocks, with each block fitted into a transport block with a CRC at the PHY layer [27]-[29].

It is expected that UMTS will deliver high-capacity mobile communications at low-costs with up to 2-Mbps data rates [30]. With the UMTS model suite, the UMTS network can be modeled to assess end-to-end throughput, service quality, drop rate, end-to-end delay, and delay jitter via a core packet network and a radio access network.

\section{THE UMTS SIMULATION MODULE}

As demonstrated in Figure 4, the simulation module of the designed UMTS comprises of a $\mathrm{CN}$, UTRAN, and user equipment (UE). CN offers switching, routing, and all functions of network management. UE has a radio interface which is provided by URTAN unit that includes the RNC and base station (Node B). The UE may be a laptop, mobile handset, or any appliance which may afford access to the network.

A simulation model can be designed for calculating the performances of QoS for voice and video transmission at UMTS network with forming an OPNET scenarios as shown in Figure 6. Here, a few nodes of UMTS can be suitable for both video as well as voice conferencing. These nodes at scenario have eminent characteristics due to their application of client-server can supported and packets are assisted on the basis of first-come-first-serve (FCFS). Many traffic modules may be considered for video simulation while for voice users, a conversational module can be concerned for utilizing. For video with low-quality, a background traffic module can be considered whereas an Interactive traffic module are considered with high video conferences.

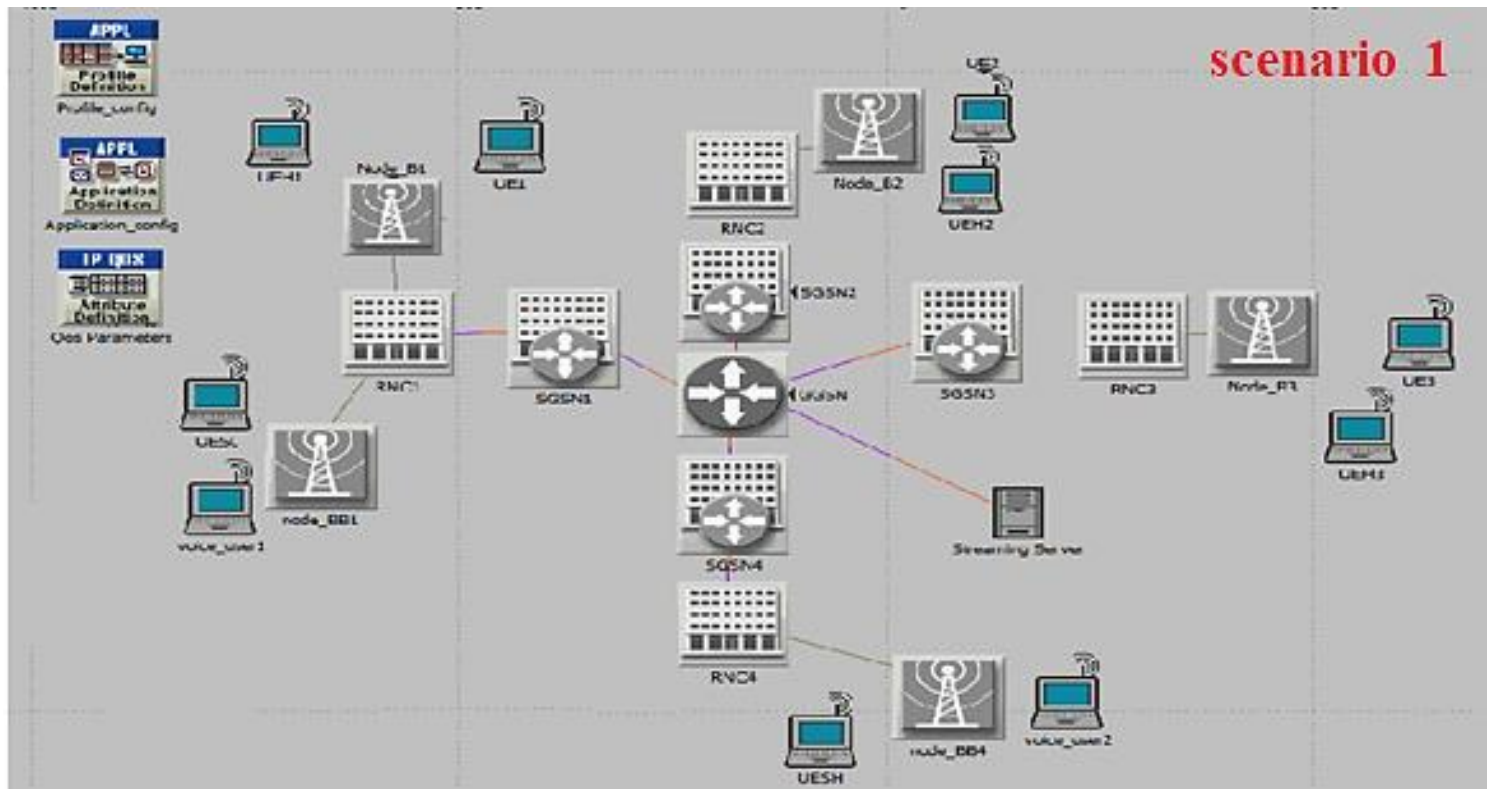

Figure 6. Scenario with QoS

During an active VoIP call from one mobile user (say, UE1) to another (say UE2), the voice packets are propagated from UE to RNC via Node-B over a Real-time Transport Protocol (RTP) embedded in the UDP. The establishment of the Radio Resource Control (RRC) connection over the channel is first ensured 
before setting up the point-to-point radio connection by the RNC. The RNC also sets up the signaling network connection before transmitting the acknowledgment to the UE. Then, the subscriber status and the accounting information are forwarded to the home AAA server for call authorization and authentication. Next, the UE will initiate the attach process before setting up the PDP context which contains the maps and routing details for packets transmitted between the UE, the SGSN, and the GGSN. The VoIP traffic in UMTS is directly routed from the Gateway to the VoIP server.

The QoS performance is estimated by introducing an IP-QoS attribute for implementation of the network's QoS. In the QoS application, different forms of queuing mechanisms exist but, in our simulation, a test of WFQ queuing schemes are applied and better performance is achieved using WFQ. It is reliable, implementation is easy and effective for explaining the traffic priority in the network. For performance optimization, differentiated service (DiffServ) may be used because it has executive control of delay, packet dropping, and bandwidth preferences. With a view to enabling DiffServ in such networks, the use of differentiated services code point (DSCP) can be applied for voice and video transmission. In the case of the QoS scenario, DSCP was applied using DiffServ to forward any packet more professionally. AF11 can be used for low-quality video conferencing and AF33 for high quality, but for video streaming, AF43 may be used and while EF is suitable for voice conferencing in the scenario as depicted in Figure 6.

\section{SIMULATION RESULTS}

The suggested model can be used for performance measurement of running data, and voice and video traffic; therefore, the received/sent traffic and throughput are the performance metrics for voice and video used with QoS and without QoS. Packet loss can be considered an important factor for determining the data transmission quality.

\subsection{Received and sent traffic data}

In Figures 7, the $\mathrm{X}$-axis denotes the simulation time (12 min) while $\mathrm{Y}$-axis denotes the received and sent data (bps). The sent data is represented by the red line while the received data is the blue line in all the plots. Figure 5 showed that there was no traffic prior to the second minute (the warm-up period). This is followed by an exponential increase in the sent and received video data until the sixth minute. After the sixth minute, the difference between the sent and received data became apparent, denoting an increase in the packet loss by $>2 \%$. This level of packet loss may be not tolerated for video conferencing.

Packet loss in a scenario with QoS during video conferencing is presented in Figure 7 (a). This figure showed an overlap between the red and blue lines due to the near equal amount of total data sent and received during video conferencing despite the high background load (90\%) at $500 \mathrm{sec}$. This suggests a lower packet loss during video conferencing in a network with QoS compared to the other scenario. At the $15^{\text {th }}$ min, the sent data was a bit higher than the received data, making it difficult to differentiate them. This represents a less than $1 \%$ packet loss which is tolerable for video conferencing (refer to Table 1).

Figure 7 (b) depicts the packet loss during voice conferencing in a network with QoS. As in Figure 7 (a), there is also a close gap between the total data sent and received during the voice conferencing, suggesting a better rate of packet loss in the network with QoS compared to the other scenario. At the $12^{\text {th }}$ min, the sent data was a bit higher than the received data, making it difficult to demarcate them. This implies an insignificant rate of packet loss $(<1 \%)$ despite the high background $(90 \%)$ load. This level of packet loss is tolerable for voice conferencing (refer to Table 1).

Table 1 UMTS QoS requirement [21]

\begin{tabular}{|c|c|c|c|c|c|c|}
\hline Medium & Applications & Symmetry Degree & Data Rate (Kbps) & \multicolumn{3}{|c|}{ Key performance parameters and target values } \\
\hline Audio & Conversational voice & Two-way & $4-25$ & $<150$, preferred $<400$ limit. & $<1$ (jitter) & $<3 \%$ FER \\
\hline Video & Video phone & Two-way & $32-384$ & $\begin{array}{l}<150, \text { preferred } \\
<400 \text { limit. } 100 \mathrm{~ms}\end{array}$ & $<1$ & $<1 \%$ FER \\
\hline
\end{tabular}

\subsection{Video throughput}

In Figures 8 (a) and (b), the simulation time $(\mathrm{min})$ is plotted in the $\mathrm{X}$-axis while the received throughput (bits/sec) is represented in the Y-axis. The throughput for the scenario with QoS is represented by the blue line in both figures while the red line represents the throughput of the scenario without QoS. The comparison of the throughput received for low-quality video conferencing in scenarios with and without QoS is presented in Figure 8 (a). This figure showed that until the $2^{\text {nd }} \min$ (the warm-up time), there was no throughput for both network scenarios, but after $2 \mathrm{~min}$, there was an equal increase in both throughputs. The significant difference in the throughputs of both scenarios increased significantly after the $6^{\text {th }}$ min. The 
throughput decreases slowly in a scenario with without QoS as the packet loss increases. The transmission throughput at the $15^{\text {th }} \mathrm{min}$ in a scenario with QoS was higher than the obtainable in the other scenario. The study showed that the packet loss affected the throughput but in this our investigation, the scenario without QoS experienced more packet loss compared to the scenario with QoS. That is the reason for the lower throughput recorded in the scenario without QoS compared to the scenario with QoS.

The throughput received by a high-quality video user in scenarios with and without QoS is compared in Figure 8 (b). Here, there was no throughput until the $2^{\text {nd }}$ min after which the scenario without QoS started showing a remarkable throughput which is a little more than that of the scenario without QoS. However, the throughput of the scenario with QoS became higher than that of the scenario without QoS after the $10^{\text {th }}$ min, implying a decrease in the throughput of the scenario without QoS after the $10^{\text {th }}$ min possibly due to the increasing rate of packet loss. However, the throughput of the scenario with QoS kept increasing consistently possibly due to adequate QoS support.

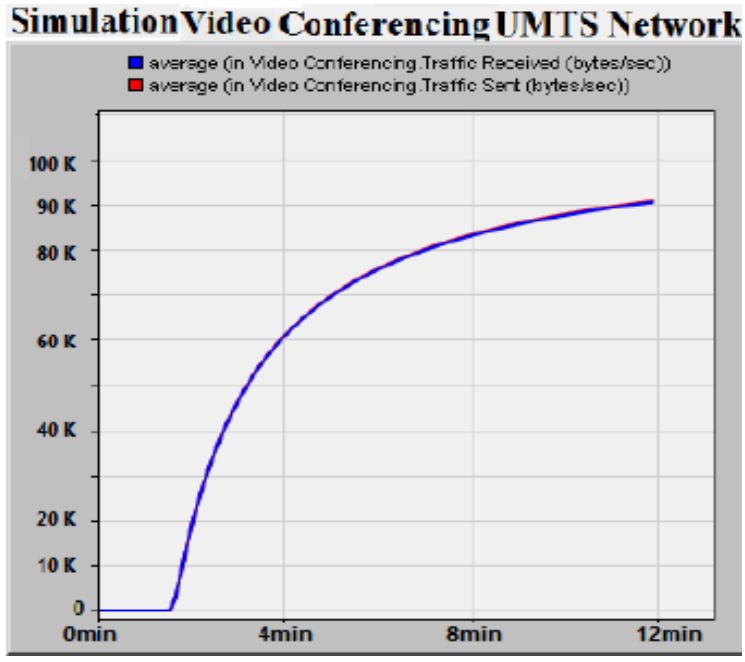

(a)

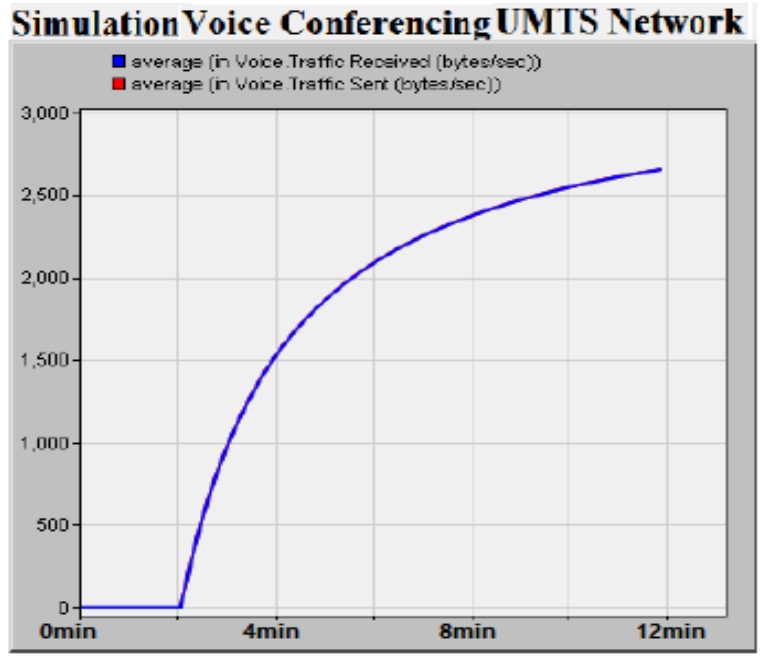

(b)

Figure 7. Show; (a) total sent and received data for video, (b) voice conferencing QoS

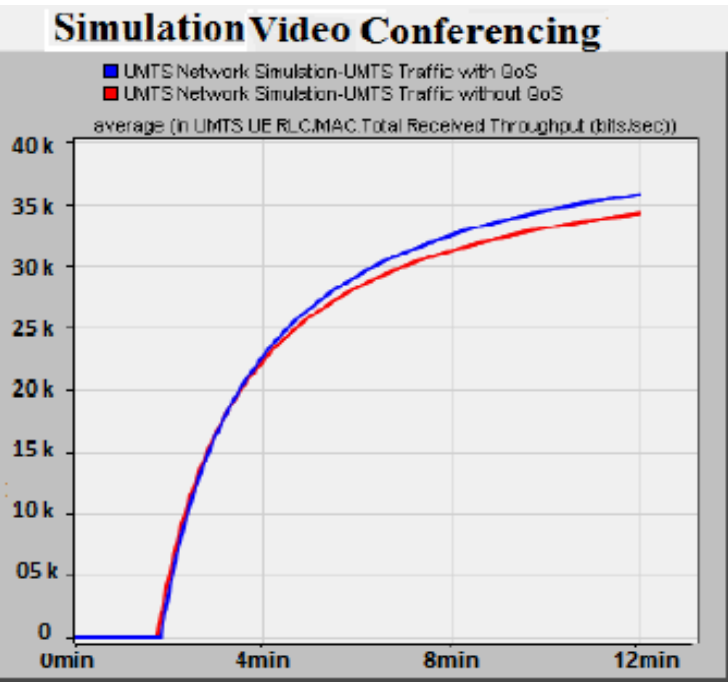

(a)

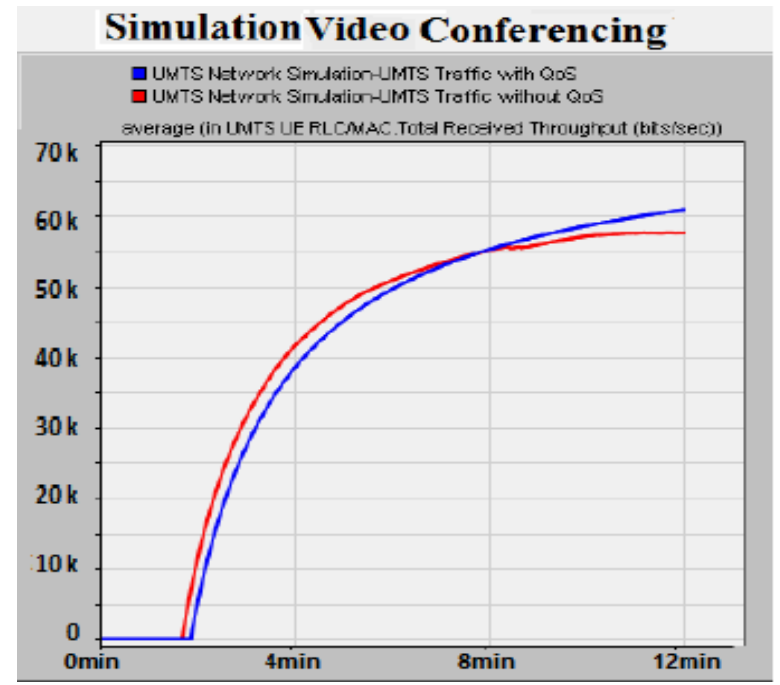

(b)

Figure 8. Throughput comparison for; (a) low quality, (b) high quality video streaming

\subsection{Voice throughput}

The throughput received by a voice user during voice conferencing in scenarios with/without QoS is compared in Figure 9. In this figure, the $\mathrm{X}$-axis is the simulation time (in min) while the received throughput 
(in bits/sec) is represented in the Y-axis. The throughput for the scenario with QoS is represented by the blue line while the red line is the throughput of the scenario without QoS. Figure 9 showed no throughput in both scenarios until the $2^{\text {nd }} \min$ (the warm-up time) but after the $2^{\text {nd }} \min$, the throughput for both scenarios increased equally until the $8^{\text {th }}$ min after which a clear difference (significant increase) was observed in the throughput between both scenarios. It was observed that the throughput at this time for the scenario without QoS was smaller than that of the QoS-aided scenario possibly due to the higher rate of packet loss in the scenario without QoS. This explains the lower throughput of the scenario without QoS compared to the scenario with QoS. Based on this figure, it can be stated that packet loss affected the throughput. Hence, a high throughput can be achieved by reducing the rate of packet loss through the use of the appropriate QoS scheme in the network.

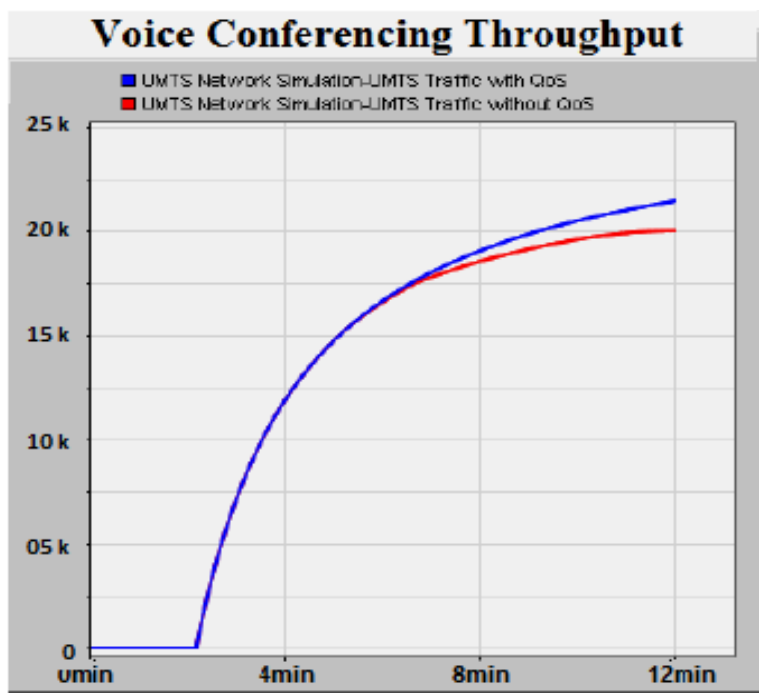

Figure 9. Comparison of throughput for voice conferencing

\section{CONCLUSION}

The compatibility of the QoS parameters with the application determines their performance. The analysis of the simulation results in this study showed that the proposed QoS scheme for the current 3G/UMTS network QoS parameters are compatible due to the appropriate suitability of QoS for both voice and video transmissions. The performance evaluation in this study was based on certain QoS parameters like the packet delay, jitter, packet loss, and throughput. The evaluation of the performance of the current QoS scheme in UMTS network was done via the analysis of the simulation results. From the analysis, it was evident that a proper QoS adaptation will ensure qualitative voice and video transmission. Therefore, the existing QoS parameters could be stated to provide appropriate support for both voice and video transmission over 3G/UMTS network.

\section{REFERENCES}

[1] S. Jadhav, H. Zhang and Z. Huang, "Performance Evaluation of Quality of VoIP in WiMAX and UMTS," 2011 12th International Conference on Parallel and Distributed Computing, Applications and Technologies, 2011, pp. 375-380, doi: 10.1109/PDCAT.2011.67.

[2] A. A. Hussien and A. H. Ali, "Comprehensive investigation of coherent optical OFDM-RoF employing 16QAM external modulation for long-haul optical communication system," International Journal Electrical and Computer Engineering (IJECE), vol. 10, no. 3, pp. 2607-2616, 2020, doi: 10.11591/ijece.v10i3.pp2607-2616.

[3] M. J. Abbas and A. A. Mahmood, "Efficiency and effectiveness video on demand over worldwide interoperability for microwave access," International Journal of Electrical and Computer Engineering (IJECE), vol. 9, no. 3, pp. 1919-1923, June 2019, doi: 10.11591/ijece.v9i3.pp1919-1923.

[4] P. A. Ochang and P. J. Irving, "Evolutionary Analysis of GSM, UMTS and LTE Mobile Network Architectures," pp. 27-39, 2016.

[5] B. M. Al-Mahadeen and A. Al-Mseden, "Improving the QoS of VoIP over WiMAX Networks Using OPNET Modeler," International Journal of Computer Science and Network Security, vol. 17, no. 8, pp. 132-142, 2017.

[6] E. A. Walelgne, J. Manner, V. Bajpai and J. Ott, "Analyzing throughput and stability in cellular networks," NOMS 2018 - 2018 IEEE/IFIP Network Operations and Management Symposium, 2018, pp. 1-9, doi: 10.1109/NOMS.2018.8406261.

Quality of service performances of video and voice transmission in universal mobile ... (Sameer A. S. Lafta) 
[7] A. T. Khalil, A. I. Abdel-Fatah and H. A. Sakr, "Rapidly IPv6 multimedia management schemes-based LTE-A wireless networks," International Journal of Electrical and Computer Engineering (IJECE), vol. 9, no. 4, pp. 30773089, 2019, doi: 10.11591/ijece.v9i4.pp3077-3089.

[8] Iskandar, I. Setyawan and H. Nuraini, "Inter-cell Interference Management Technique for Multi-Cell LTE-A Network," International Journal of Electrical and Computer Engineering (IJECE), vol. 7, no. 5, p. 2696, 2017, doi: 10.11591/ijece.v7i5.pp2696-2705.

[9] A. Farhood, M. K. Naji, S. H. R. H. Rhaif and A. Ali, "Design and analysis of dual band integrated hexagonal shaped microstrip UWB antenna," Indonesian Journal Electrical Engineering and Computer Science (IJEECS), vol. 15, no. 1, pp. 294-299, 2019, doi: 10.11591/ijeecs.v15.i1.pp294-299.

[10] Z. Li and J. Yang, "Investigation on Handover in WiMAX and Performance Comparison over WiMAX and LTE," International Research Journal of Engineering and Technology (IRJET), vol. 3, no. 5, pp. 15-24, 2016.

[11] S. I. Zatega, A. Lipovac and V. Lipovac, "Logistic regression based in-service assessment of mobile web browsing service quality acceptability," EURASIP Journal on Wireless Communications and Networking, vol. 2020, pp. 121, 2020, doi: 10.1186/s13638-020-01708-2

[12] H. Ashamisi, H. S. Alshamsi, I. Kostanic and J. Zec, "An Efficient approach for evaluating performance of LTE network using android application in the smart-phones," Mobile comunication, 2018, doi: 10.9790/96220804027983.

[13] S. Narejo, F. Kulsoom and M. M. Jawaid, "Performance Evaluation of Interactive Video Streaming over WiMAX Network," International Journal of Electrical and Computer Engineering (IJECE), vol. 7, no. 1, pp. 344-352, 2017, doi: 10.11591/ijece.v7i1.pp344-352.

[14] A. L. Karthika, M. G. Sumithra and A. Shanmugam, "Performance of voice in integrated WiMAX-WLAN and UMTS-WLAN," International Journal of Innovative Research in Science, Engineering and Technology, vol. 2, no. 4, 2013.

[15] M. K. Banjanin, M Stojčić, D. Drajić, Z. Ćurguz, Z. Milanović, A. Stjepanović, "Adaptive Modeling of Prediction of Telecommunications Network Throughput Performances in the Domain of Motorway Coverage," Applied Sciences, vol. 11, no. 8, id: 3559. 2021, doi: 10.3390/app11083559

[16] S. A. Lafta, A. Hasan Ali, M. M. Kareem, Y. A. Hussein and A. H. Ali, "Performance simulation of broadband multimedia wireless networks simulation based on OPNET," Indonesian Journal Electrical Engineering and Computer Science (IJEECS), vol. 17, no. 1, pp. 1-9, 2020, doi: 10.11591/ijeecs.v17.i1.pp1-9.

[17] A. Samba, Y. Busnel, A. Blanc, P. Dooze and G. Simon, "Instantaneous throughput prediction in cellular networks: Which information is needed?," 2017 IFIP/IEEE Symposium on Integrated Network and Service Management (IM), 2017, pp. 624-627, doi: 10.23919/INM.2017.7987345.

[18] G. Vijayalakshmy and G. Sivaradje, "WiMAX-UMTS Converging Architecture with IMS Signaling analysis to achieve QoS," Procedia Technology, vol. 6, pp. 16-23, 2012, doi: 10.1016/j.protcy.2012.10.003.

[19] H. M. Noman, A. A. Abdulrazzaq, M. M. Kareem and A. H. Ali, "Improvement Investigation of the TCP Algorithms with Avoiding Network Congestion Based on OPNET," 2nd ICSET 2019, IOP Conf. Series: Materials Science and Engineering (2019), vol. 518, no. 5, p. 052025, 2019.

[20] V. Bharathi and L. Nithyanandan, "Efficient Cooperative Relaying in UMTS-WiMAX-WLAN Overlaid Heterogeneous Networks," Elsevier Processing of International Conference on Advances in Communication, Network and Computing, CNC, 2014.

[21] M. M. Kareem, M. Ismail, M. A. Altahrawi, N. Arsad, M. F. Mansor and A. H. Ali, "Grid Based Clustering Technique in Wireless Sensor Network using Hierarchical Routing Protocol," 2018 IEEE 4th International Symposium on Telecommunication Technologies (ISTT), 2018, pp. 1-5, doi: 10.1109/ISTT.2018.8701720.

[22] Chuah, Mooi Choo, Zhang and Qinqing, "Design and Performance of 3G Wireless Networks and wireless LANS," Springer Science-l-Business Media, Inc., 2006.

[23] C. Bouras, A Papazois and A. Alexiou, "Congestion Control for Multicast Transmission over UMTS," in Wireless Quality of Service, Dr. Yan Zhang, Ed. Norway: Auerbach, 2009, ch. 10, pp. 291-311.

[24] M. J. Mnati, R. F. Chisab, A. M. Al-Rawi, A. H. Ali and A. V.den Bossche, "An open-source non-contact thermometer using low-cost electronic components," HardwareX, vol. 9, p. e00183, 2021, doi: 10.1016/j.ohx.2021.e00183.

[25] Y. Du, Fung P. Tso and W. Jia, "Architecture design of video transmission between UMTS and WSN," 2008 First IEEE International Conference on Ubi-Media Computing, 2008, pp. 57-62, doi: 10.1109/UMEDIA.2008.4570866.

[26] G. Putnik, L. Ferreira, N. Lopes and Z. Putnik, "What is a Cyber-Physical System: Definitions and Models Spectrum," FME Transactions, vol. 47, no. 4, pp. 663-674, 2019, doi: 10.5937/fmet1904663P.

[27] S. A. Lonkar and K. T. V. Reddy, "Analysis of audio and video quality of voice over LTE (VoLTE) call," International Journal of Information Technology, vol. 2020, pp. 1-14, 2020, doi: 10.1007/s41870-020-00455-3.

[28] T. ur Rehman, M. A. I. Baig and A. Ahmad, "LTE downlink throughput modeling using neural networks," 2017 IEEE 8th Annual Ubiquitous Computing, Electronics and Mobile Communication Conference (UEMCON), 2017, pp. 265-270, doi: 10.1109/UEMCON.2017.8249044.

[29] A. Sadeghi, M. Luvisotto, F. Lahouti and M. Zorzi, "Analysis of Statistical QoS in Half Duplex and Full Duplex Dense Heterogeneous Cellular Networks," arXiv preprint arXiv:1708.06215, 2017.

[30] A. Perez, "Implementing IP and Ethernet on the 4G Mobile Network," Elsevier, 2017. 